\title{
Mass Spectrometric Analysis of Multiple Pertussis Toxins and Toxoids
}

\author{
Yulanda M. Williamson, ${ }^{1}$ Hercules Moura, ${ }^{1}$ David Schieltz, ${ }^{1}$ Jon Rees, ${ }^{1}$ Adrian R. Woolfitt, ${ }^{1}$ \\ James L. Pirkle, ${ }^{1}$ Jacquelyn S. Sampson, ${ }^{2}$ Maria L. Tondella, ${ }^{2}$ Edwin Ades, ${ }^{2}$ George Carlone, ${ }^{2}$ \\ and John R. Barr ${ }^{1}$ \\ ${ }^{1}$ Biological Mass Spectrometry Laboratory, National Center for Environmental Health, Centers for Disease Control and Prevention, \\ Chamblee, GA 30341, USA \\ ${ }^{2}$ Division of Bacterial Diseases, National Center for Immunizations and Respiratory Diseases, \\ Centers for Disease Control and Prevention, Atlanta, GA 30333, USA
}

Correspondence should be addressed to John R. Barr, jbarr@cdc.gov

Received 15 December 2009; Accepted 9 March 2010

Academic Editor: Yongqun Oliver He

Copyright (C) 2010 Yulanda M. Williamson et al. This is an open access article distributed under the Creative Commons Attribution License, which permits unrestricted use, distribution, and reproduction in any medium, provided the original work is properly cited.

Bordetella pertussis (Bp) is the causative agent of pertussis, a vaccine preventable disease occurring primarily in children. In recent years, there has been increased reporting of pertussis. Current pertussis vaccines are acellular and consist of Bp proteins including the major virulence factor pertussis toxin (Ptx), a 5-subunit exotoxin. Variation in Ptx subunit amino acid (AA) sequence could possibly affect the immune response. A blind comparative mass spectrometric (MS) analysis of commercially available Ptx as well as the chemically modified toxoid (Ptxd) from licensed vaccines was performed to assess peptide sequence and AA coverage variability as well as relative amounts of Ptx subunits. Qualitatively, there are similarities among the various sources based on AA percent coverages and MS/MS fragmentation profiles. Additionally, based on a label-free mass spectrometry-based quantification method there is differential relative abundance of the subunits among the sources.

\section{Introduction}

Bordetella pertussis (Bp), a gram-negative bacterium, is the causative agent of pertussis, commonly known as whooping cough [1]. Pertussis is a highly communicable respiratory infection that in the absence of vaccination primarily afflicts infants and children. Transmission occurs via direct contact with discharges from respiratory mucous membranes of infected persons. Persons who contract pertussis develop a persistent cough that includes the characteristic highpitched "whoop" sound that can last up to eight weeks $[1,2]$. Pertussis is a vaccine preventable disease but according to the World Health Organization (WHO), there are an estimated 50 million cases of pertussis per year worldwide with approximately 300,000 leading to death $[3,4]$. The majority of the high mortality rates come from developing countries. In the United States acellular pertussis vaccines are administered to infants and young children [5] and in 2006 were recommended for adolescents and adults [68]. Acellular vaccines consist of up to five Bp components including the major virulence factor pertussis toxin (Ptx), filamentous hemagluttinin, pertactin, fimbrae 2, and/or fimbrae 3 [3]. Pertussis vaccines are generally formulated in combination with vaccine immunogens for other diseases, such as tetanus and diphtheria [9].

Ptx, a five-subunit protein complex (S1-S5) is a major bacterial exotoxin, surface adhesin, and porin, is involved in the establishment of infection in humans and has been shown to be an important component of the vaccine [10]. Ptx, a $\sim 105 \mathrm{kDa} A-B$ protein complex, comprises the protomer (A) and oligomer (B). The A component consists of $\mathrm{S} 1(\sim 22 \mathrm{kDa})$, which contains ADP ribosylation enzymatic activity and is the most immunoreactive region of the toxin. The B portion consists of S2, S3, S4, and S5, decreasing in size from $\sim 20 \mathrm{kDa}$ to $10 \mathrm{kDa}$, respectively, and is associated with adherence to human host cells $[11,12]$. 
From a cellular perspective, the B portion binds to cells inducing the enzymatic activity of $\mathrm{S} 1$ and its transversion into the cell, followed by inactivation of $\mathrm{G}$ proteins by $\mathrm{S} 1$ and subsequent initiation of an intracellular cascade of signaling events which includes factors that regulate the immune response [11]. Importantly, the Ptxs in current vaccines have been inactivated (toxoids; Ptxd) by covalent crosslinkages formed using agents such as formaldehyde or glutaraldehyde $[13,14]$.

Recently, the U.S. and several other countries have reported an increase in pertussis cases. Factors such as waning immunity after natural infection or immunization, improved molecular-based diagnostics, increased surveillance, or changes in circulating strain antigenicity have all been hypothesized as contributing factors [3] and have led researchers to re-examine pertussis vaccine components and preparation strategies. Changes in Ptx subunit protein sequences as well as the amounts present in the vaccine formulation could affect the overall immune response. Moreover, identification of commonalities as well as potential disparities of various Ptx and Ptxd sources on a proteomic level could provide insight into changes in population immune responses to the vaccine.

Mass spectrometry (MS), a powerful and sensitive analytical tool has been used to identify, differentiate, and characterize proteins in complex mixtures [15]. In particular, MS techniques, such as liquid chromatographyelectrospray ionization (LC-ESI) tandem MS (MS/MS) or matrix-assisted laser desorption ionization time-of-flight (MALDI-TOF) MS/MS, which vary in their rapidity and sensitivity of peptide detection as a result of enzymatic cleavage, are commonly used in proteomic analysis $[15,16]$. Proteins can be proteolytically cleaved by enzymes such as trypsin and the peptides generated are ionized and separated based on their mass-to-charge ratios. The peptides and the proteins, from which they originated, are identified based on comparisons between the MS/MS fragmentation patterns and protein databases. [17]. For instance, Bottero et al. used MALDI-TOF MS/MS as a means to characterize enriched Bp membrane proteins, which included the Ptx S1, from different strains [18]. Alternatively, Tumala and colleagues have used liquid chromatography MS/MS (LC-MS/MS) to accurately measure the molecular weights of Ptx subunits as well as to identify novel chemical modification sites [19].

In this study, a blind comparative mass spectrometric analysis of Ptx and Ptxd from multiple sources was performed to assess potential sequence and amino acid (AA) coverage variability as well as relative amounts of pertussis toxin subunits. This first-phase study demonstrates the sensitivity of mass spectrometry in ascertaining differences at the peptide sequence level and may provide a basis for future correlative immunoproteomic studies.

\section{Methods}

2.1. Pertussis Toxin and Toxoid Sources. The commercially available Ptx and Ptxd were purchased from Protein Express, Inc. (Cincinnati, OH, USA), LIST Biologicals, Inc. (Campbell, CA, USA), Sanofi Pasteur Pharmaceutical (Paris,
France), or GSK Pharmaceutical (London, UK). The sources were blindly assigned a letter, in which Ptxs are represented as A or B, while the chemically inactivated Ptxd samples are denoted as $\mathrm{C}$ or $\mathrm{D}$.

2.2. Pertussis Toxin and Toxoid Digestions. The Ptx and Ptxd sources $(10 \mu \mathrm{g})$ were vortexed and precipitated with $100 \%$ acetonitrile $(\mathrm{ACN})$, for 60 minutes, and then dried using a vacuum centrifuge. Trypsin ( $5 \mu \mathrm{g})$ (Promega Corporation, Madison, WI, USA) was added and the volume was brought to $100 \mu \mathrm{L}$ with tryptic digestion buffer $(50 \mathrm{mM}$ ammonium bicarbonate adjusted to $\mathrm{pH} 8.5$ with $\mathrm{NaOH}, 0.6 \mathrm{mM} \mathrm{CaCl}_{2}$, $0.1 \%(\mathrm{w} / \mathrm{v})$ potassium azide $\left(\mathrm{KN}_{3}\right)$, and $3 \mathrm{mg} / \mathrm{L}$ phenol red). The samples were incubated at $37^{\circ} \mathrm{C}$ for 3,6 , and 24 hours. Digested samples (250 ng total) were subsequently prepared for either MALDI-TOF MS/MS or LC-MS/MS. Additionally, in separate experiments, samples were treated with an acid cleavable detergent, RapiGest (RG; Waters Corporation, Milford, MA, USA) to assess the efficiency of tryptic digestion. $0.1 \%$ RG in tryptic digestion buffer was added to Ptx or Ptxd $(10 \mu \mathrm{g})$ samples. The samples were next incubated at $100^{\circ} \mathrm{C}$ for 5 minutes followed by cooling at room temperature with the subsequent addition of trypsin $(5 \mu \mathrm{g})$. Trypsin digestions were performed as stated above. However, prior to MS analysis, the RG was inactivated by cleavage with $0.175 \mathrm{M} \mathrm{HCL}$ at $37^{\circ} \mathrm{C}$ for 30 minutes, and the supernatant (obtained by centrifugation for 15 minutes at $17,300 \mathrm{~g}$ ) was transferred to a new vial.

2.3. MS Sample Preparation. Samples for MALDI-TOF MS/MS were resuspended in an equal volume of $0.1 \%$ trifluoroacetic acid (TFA), and the peptides were separated using a $\mathrm{C}_{18}$ zip-tip minicolumn (Millipore Corporation, Billerica, MA, USA) and eluted in two steps with $3 \mu \mathrm{L}$ of $30 \% \mathrm{ACN}$ in $0.1 \%$ TFA and then $3 \mu \mathrm{L}$ of $80 \% \mathrm{ACN}$ in $0.1 \%$ TFA. The eluates were mixed with $10 \mu \mathrm{L} \alpha$-cyano-4hydroxycinnamic acid ( $\alpha$-CHCA) (Sigma Aldrich, St. Louis, MO, USA) and $0.5 \mu \mathrm{L}$ was next spotted on to a stainless steel plate. A protein mix diluted at $1: 10$ was spotted on the plate in the appropriate wells and used for instrument calibration purposes. For LC-MS/MS, samples were simply resuspended in equal volumes of $0.1 \%$ formic acid (FA).

\subsection{Mass Spectrometry}

2.4.1. MALDI-TOF MS/MS. Mass spectra of each sample well were obtained by scanning from 800 to $4000 \mathrm{~m} / \mathrm{z}$ in MS positive-ion reflector mode on the Applied Biosystems 4800 Proteomics Analyzer (Framingham, MA, USA). The instrument uses a nd:YAG laser at $337 \mathrm{~nm}$ with a $200 \mathrm{MHz}$ repetition rate, and each spectrum is an average of 1000 laser shots. In MS/MS mode, the CID spectra were generated using air as the collision gas at $1 \mathrm{kV}$ and an average of 500 laser shots for MS/MS acquisition. The instrument was tuned and calibrated for MS analysis using a mixture of five peptides: des-Arg1-Bradykinin (m/z 904.47), angiotensin I (m/z 1,296.69), Glu1-fibrinopeptide B (m/z 1,570.68), ACTH $(1-17)(\mathrm{m} / \mathrm{z} 2093.08)$, and ACTH (1839)(m/z 2,465.20). For MS/MS mass calibration, known 
fragment ion masses observed in the Glu1-fibrinopeptide B were used. The instrument was programmed for automated MS/MS acquisition using data dependent acquisition (DDA) mode. Selected peptides were subjected to MS/MS analysis to confirm their identity through sequence determination.

All MS/MS spectra were automatically submitted to a Mascot Server through Mascot Distiller (Matrix Science Inc., London, UK; version 2.2.1.0) and searched against the entire NCBInr database or a modified NCBInr database created to search "Bordetella" or "pertussis" proteins with deamidation, oxidation and carbamidomethylation assigned as variable modifications. Tryptic constraints were applied, allowing up to two missed cleavages. Peptide searches were performed with an initial tolerance on mass measurement of $100 \mathrm{ppm}$ in MS mode and $150 \mathrm{ppm}$ in MS/MS mode. All matched peptides were verified manually and the searches combined and processed by Scaffold (Proteome Software Inc., Portland, OR, USA; version 2.03.01).

2.4.2. Nano-LC ESI-MS/MS (LTQ-Orbitrap). Protein identification was achieved by using nanoflow liquid chromatography, data-dependant tandem mass spectrometry, and database searching. A $365 \mu \mathrm{m}$ by $75 \mu \mathrm{m}$ fused silica pulled needle capillary (New Objective, Inc., Woburn, MA) was filled in house with $10 \mathrm{~cm}$ of $5 \mu \mathrm{m}$ Symmetry 300 reverse phase packing material (Waters Inc., Bedford, MA). Protein digests were directly loaded onto the analytical column without using a trap column and introduced using an Eksigent autosampler. The gradient was delivered by an Eksigent 2D nanoLC system (Eksigent Technologies, Inc, Dublin, CA) where the mobile phase solvents consisted of: (solvent A) $0.2 \%$ formic acid (Thermo Scientific, Rockford, IL), $0.005 \%$ trifluoroacetic acid (Sigma-Aldrich) in water (Burdick and Jackson, Muskegon, MI), (solvent B) $0.2 \%$ formic acid, and $0.005 \%$ trifluoroacetic acid in acetonitrile (Burdick and Jackson). The gradient flow was set at $400 \mathrm{~nL} / \mathrm{min}$, and the profile consisted of a hold at $5 \% \mathrm{~B}$ for 5 minutes followed by a ramp to $30 \%$ B over 100 minutes then a ramp up to $90 \%$ B in 5 minutes and a hold at $90 \%$ for 2 minutes before returning to $5 \% \mathrm{~B}$ in 2 minutes and re-equilibration at $5 \% \mathrm{~B}$ for 20 minutes. Peptides were nano-electrosprayed into an LTQ Orbitrap tandem mass spectrometer (Thermo Scientific, San Jose, CA). The voltage applied to the nano-electrospray source was $2.0 \mathrm{kV}$. The mass spectrometer was programmed to perform data-dependant acquisition by scanning the mass range from $\mathrm{m} / \mathrm{z} 400$ to 1600 at a nominal resolution setting of 60,000 for parent ion acquisition in the Orbitrap. Then tandem mass spectra of doubly-charged and higher-charge states were acquired for the top 10 most intense ions. Singlycharged ions were not considered for MS/MS. All tandem mass spectra were recorded using the linear ion trap. This DDA process cycled continuously throughout the duration of the gradient.

All tandem mass spectra were extracted from the raw data file using Mascot Distiller (Matrix Science, London, UK; version 2.2.1.0) and searched using Mascot (version 2.2.0). Mascot was setup to search using the entire NCBInr database or a modified NCBInr database created to search "Bordetella" or "pertussis" recognized proteins in which trypsin is used as the digestion agent. Mascot was searched with a fragment ion tolerance mass of $0.80 \mathrm{Da}$ and a parent ion tolerance of $200 \mathrm{ppm}$. Variable modifications for the mascot search were deamidation, oxidation, and carbamidomethylation. Scaffold was used to validate MS/MS-based peptide and protein identifications. Peptide identifications were accepted if they could be established at greater than $95.0 \%$ probability as specified by the Peptide Prophet algorithm [20]. Protein identifications were accepted if they could be established at greater than $99.0 \%$ probability and contained at least 2 identified peptides [21]. With these stringent parameters of Peptide Prophet and Protein Prophet, the false discovery rate was zero.

\subsubsection{Nano-LC ESI-MS/MS (Q-Tof Premier). LC-MS/MS} was carried out using a nanoAcquity ultra-performance liquid chromatography (UPLC) coupled to a QTof Premier MS system (Waters Corporation). LC separation was performed using a Symmetry $\mathrm{C}_{18}$ trapping column and a $\mathrm{BEH} \mathrm{C}_{18}$ column $(100 \mu \mathrm{m}$ I.D. $\times 100 \mathrm{~mm}$ long with $1.7 \mu \mathrm{m}$ packing $)$, at a flow rate of $1.2 \mu \mathrm{L} / \mathrm{min}$. Standard water/ACN gradients containing $0.1 \%$ formic acid in both solvents were used for elution, with the ACN increasing linearly from $1 \%$ to $50 \%$ in 50 minutes, and an overall cycle time of 90 minutes. The QTof utilized an MS ${ }^{\mathrm{E}}$ (or Protein Expression) method, which involved acquiring data-independent alternating lowand high-energy scans over the $\mathrm{m} / \mathrm{z}$ range $50-1990$ in 0.6 second, along with lockmass data on a separate channel to obtain accurate mass measurement. The data obtained using the $\mathrm{MS}^{\mathrm{E}}$ method were further processed using ProteinLynx Global Server v2.3, which provided statistically validated peptide and protein identification using a SwissProt-Trembl protein database, along with relative protein quantification analysis. Root mean square mass accuracies were typically within $8 \mathrm{ppm}$ for the MS data, and within $15 \mathrm{ppm}$ for MS/MS data. Also the false positive rate was $4 \%$. Lastly, the relative quantification assessed in this study used an internal standard from a known protein and is considered a surrogate.

\section{Results}

A mass spectrometric analysis was performed to assess protein sequence homogeneity of Ptx and Ptxd. MALDI-TOF $\mathrm{MS} / \mathrm{MS}$, and LC-MS/MS approaches revealed comparable to varying AA percent composition profiles (percent is based on the number of AA (within tryptic peptides) generated divided by the total number of AA in the protein) among Ptx and Ptxd (Table 1) as well as a compilation of detected peptides (Table 2). Generally, Ptx A and B digested with trypsin generated higher AA coverages among the nontreated oligomeric subunits compared to their chemically modified Ptxd counterparts regardless of the MS system used. Additionally, trypsin digests of Ptx B generated the highest average among S2-S5 compared to all samples. Also, for both Ptxd samples there was low to no detection of S2-S5 peptides.

However, AA coverage reports for S1 on average between Ptx and Ptxd were fairly comparable with 45 and $44 \%$, respectively. Probing further, LC-MS/MS instruments 


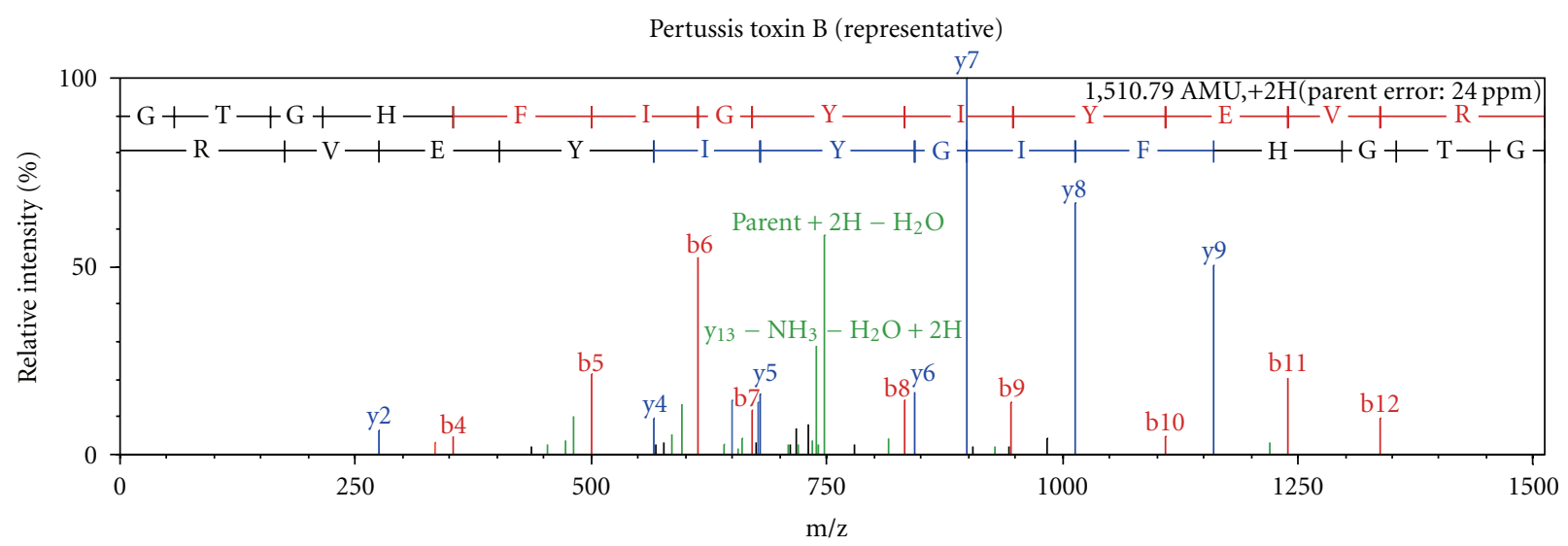

(a)

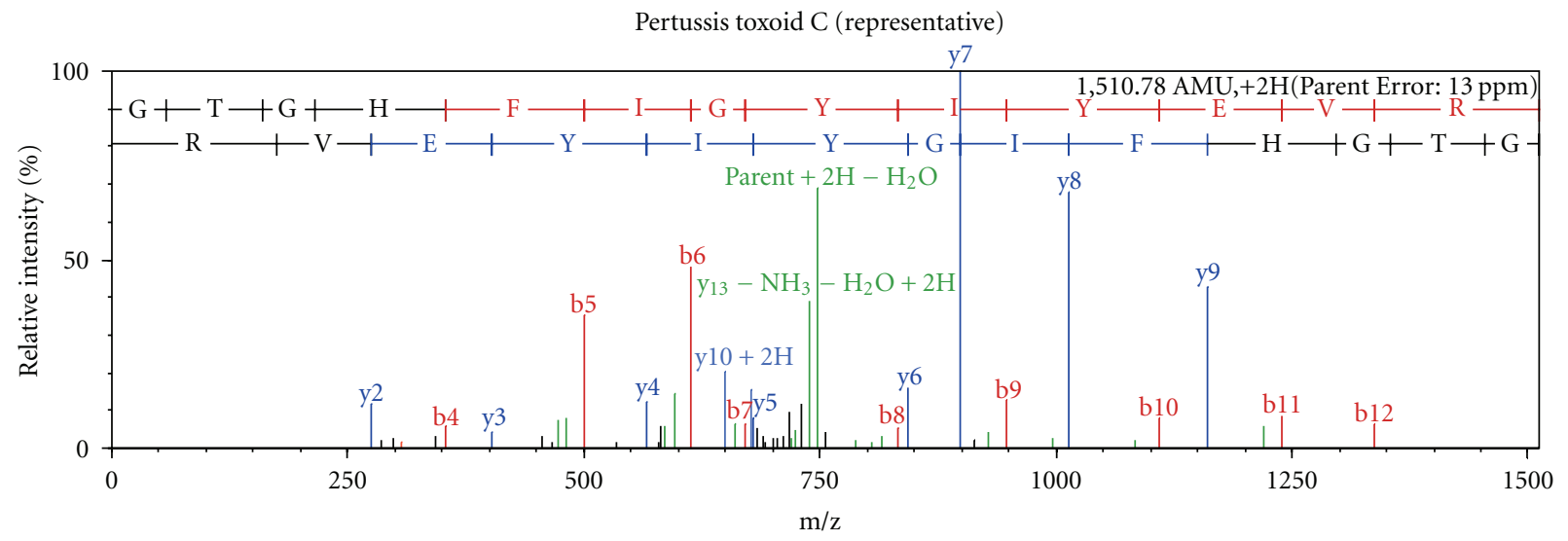

(b)

FIGURE 1: MS/MS spectra illustrating similar fragmentation profiles of Subunit 1 peptide GTGHFIGIYEVR from representative pertussis toxin (B) and pertussis toxoid (C). Nano Liquid Chromatography MS/MS were performed using the LTQ-Orbitrap on tryptic peptides obtained from pertussis toxin B and pertussis toxoid C. The MS/MS data was searched against the NCBI database and the peptide spectrum was analyzed [comparing relative peak intensity (0-100\%) versus mass/charge (0 to 1500)] using the Scaffold software. The red and blue lines represent $\mathrm{y}$ - and $\mathrm{b}$-ion distribution peaks, respectively.

TABLE 1: Comparative toxin and toxoid sequence analysis ( $\%$ amino acid coverage).

\begin{tabular}{ccccccccccccc}
\hline & A-1 & A-2 & A-3 & B-1 & B-2 & B-3 & C-1 & C-2 & C-3 & D-1 & D-2 & D-3 \\
\hline S1 & 30 & 58 & 26 & 35 & 69 & 55 & 36 & 49 & 61 & 23 & 41 & 58 \\
S2 & 6 & 33 & 28 & 20 & 39 & 31 & ND & 14 & 16 & ND & 5 & 19 \\
S3 ND & 28 & 18 & 18 & 39 & 49 & ND & 12 & 22 & ND & 18 & 20 \\
S4 & 20 & 53 & 13 & 41 & 57 & 42 & ND & 28 & ND & ND & 28 & ND \\
S5 ND & ND & 8 & ND & ND & 8 & ND & ND & ND & ND & ND & 8 \\
\hline
\end{tabular}

-ND: Not Detected

-Pertussis toxin Subunits (S1-S5)

-Sources: Pertussis toxins (A and B) and Pertussis toxoids (C and D)

-Instruments: 1-MALDI-TOF MS (4800 Proteomics Analyzer), 2-ESI-MS

(LTQ-Orbitrap), and 3-ESI-MS (Q-Tof)

proved to be more fruitful in terms of yielding a highersequence coverage and an increase in peptide detection. Specifically, S1 gave a higher-percent coverage by LCMS/MS instruments resulting in a 1.7 -fold higher-overall average as compared to the MALDI-TOF mass-spectrometric approach.

Tryptic digestions supplemented with RG resulted in an increase in sequence coverage in all samples and differentially within the subunits (Table 3 ). RG had a greater effect on the Ptxd giving rise to the greatest increase in sequence coverage. Ptxd D (2.2) resulted in a slightly greater overall fold change as compared to Ptxd C (1.6), in the presence of RG, while fold changes between all subunits were lower in both Ptx A (0.5) and Ptx B (1.3). Collectively for both Ptx and Ptxd, S1, S3, and S5 had marked gains in the presence of RG with a nearly $1.5,2.5$, and 10 -fold increase, respectively.

As the sequence coverage increased in the presence of RG, the number of peptides detected by LC-MS/MS increased as well and to a lesser extent in MALDI-TOF (data not shown). Since the LC-MS/MS LTQ Orbitrap instrument gave the optimum sequence coverage (in particular for the toxins) in the presence of RG, a comparative assessment of peptide detection for Ptx subunits among all sources was compiled (Table 4). Table 4 reveals common and unique 
TABLE 2: Mass spectrometry-detected peptides found among all pertussis toxins and toxoids.

\begin{tabular}{|c|c|c|c|c|c|}
\hline & Peptides Detected & Toxin A & Toxin B & Toxoid C & Toxoid D \\
\hline \multirow[t]{13}{*}{ S1 } & DDPPATVYR (35-43) & $\mathrm{X}$ & $\mathrm{X}$ & & $\mathrm{X}$ \\
\hline & SCQVGSSNSAFVSTSSSR (74-91) & & $\mathrm{X}$ & $\mathrm{X}$ & \\
\hline & YTEVYLEHR (93-101) & $\mathrm{X}$ & $\mathrm{X}$ & $\mathrm{X}$ & $\mathrm{X}$ \\
\hline & MQEAVEAER (102-110) & $\mathrm{X}$ & $\mathrm{X}$ & $\mathrm{X}$ & $\mathrm{X}$ \\
\hline & GTGHFIGYIYEVR (114-126) & $\mathrm{X}$ & $\mathrm{X}$ & $\mathrm{X}$ & $\mathrm{X}$ \\
\hline & ADNNFYGAASSYFEYVDTYGDNAGR (127-151) & $\mathrm{X}$ & $\mathrm{X}$ & $\mathrm{X}$ & $\mathrm{X}$ \\
\hline & ILAGALATYQSEYLAHR (152-168) & $\mathrm{X}$ & $\mathrm{X}$ & $\mathrm{X}$ & $\mathrm{X}$ \\
\hline & IPPENIR (170-176) & $\mathrm{X}$ & $\mathrm{X}$ & $\mathrm{X}$ & $\mathrm{X}$ \\
\hline & VYHNGITGETTTTEYSNAR (181-199) & $\mathrm{X}$ & $\mathrm{X}$ & $\mathrm{X}$ & \\
\hline & YVSQQTRANPNYTSR (200-215) & & & $\mathrm{X}$ & \\
\hline & ANPNPYTSR (207-215) & & $\mathrm{X}$ & & \\
\hline & SVASIVGTLVR (217-227) & $\mathrm{X}$ & $\mathrm{X}$ & $\mathrm{X}$ & $\mathrm{X}$ \\
\hline & QAESSEAMAAWSER (239-252) & $\mathrm{X}$ & $\mathrm{X}$ & $\mathrm{X}$ & $\mathrm{X}$ \\
\hline \multirow[t]{8}{*}{$\mathrm{S} 2$} & ALTVAELR (56-63) & $\mathrm{X}$ & & $\mathrm{X}$ & $\mathrm{X}$ \\
\hline & GSGDLQEYLR (64-73) & $\mathrm{X}$ & $\mathrm{X}$ & $\mathrm{X}$ & $\mathrm{X}$ \\
\hline & GWSIFALYDGTYLGGEYGGVIK (78-99) & $\mathrm{X}$ & $\mathrm{X}$ & & \\
\hline & DGTPGGAFDLK (100-110) & $\mathrm{X}$ & $\mathrm{X}$ & $\mathrm{X}$ & $\mathrm{X}$ \\
\hline & NTGQPATDHYYSNVTATR (120-137) & $\mathrm{X}$ & $\mathrm{X}$ & $\mathrm{X}$ & \\
\hline & LCAVFVR (146-152) & & & & $\mathrm{X}$ \\
\hline & SGQPVIGACTSPYDGK (153-168) & $\mathrm{X}$ & $\mathrm{X}$ & $\mathrm{X}$ & \\
\hline & MLYLIYVAGISVR (179-191) & $\mathrm{X}$ & & & \\
\hline \multirow[t]{12}{*}{ S3 } & ALFTQQGGAYGR (39-50) & $\mathrm{X}$ & $\mathrm{X}$ & $\mathrm{X}$ & $\mathrm{X}$ \\
\hline & ALTVAELR (57-64) & $\mathrm{X}$ & & $\mathrm{X}$ & $\mathrm{X}$ \\
\hline & GNAELQTYLR (65-74) & $\mathrm{X}$ & & $\mathrm{X}$ & $\mathrm{X}$ \\
\hline & QITPGWSIYGLYDGTYLGQAYGGIIK (75-100) & & $\mathrm{X}$ & $\mathrm{X}$ & $\mathrm{X}$ \\
\hline & DAPPGAGFIYR (101-111) & $\mathrm{X}$ & $\mathrm{X}$ & $\mathrm{X}$ & $\mathrm{X}$ \\
\hline & ETFCITTIYK (112-121) & $\mathrm{X}$ & $\mathrm{X}$ & $\mathrm{X}$ & $\mathrm{X}$ \\
\hline & TGQPAADHYYSK (122-133) & $\mathrm{X}$ & $\mathrm{X}$ & $\mathrm{X}$ & \\
\hline & LLASTNSR (139-146) & & & & $\mathrm{X}$ \\
\hline & LCAVFVR (147-153) & & & $\mathrm{X}$ & \\
\hline & DGQSVIGACASPYEGR (154-169) & $\mathrm{X}$ & & & \\
\hline & DMYDALR (172-178) & & $\mathrm{X}$ & & \\
\hline & LLYMIYMSGLAVR (180-192) & $\mathrm{X}$ & $\mathrm{X}$ & & \\
\hline \multirow[t]{6}{*}{ S4 } & DVPYVLVK (43-50) & $\mathrm{X}$ & & & \\
\hline & TNMVVTSVAMKPYEVTPTR (51-69) & $\mathrm{X}$ & $\mathrm{X}$ & $\mathrm{X}$ & $\mathrm{X}$ \\
\hline & MLVCGIAAK (70-78) & & $\mathrm{X}$ & & \\
\hline & LGAAASSPDAHVPFCFGK (79-96) & $\mathrm{X}$ & $\mathrm{X}$ & & \\
\hline & RPGSSPMEVMLR (100-111) & $\mathrm{X}$ & $\mathrm{X}$ & $\mathrm{X}$ & $\mathrm{X}$ \\
\hline & QLTFEGKPALELIR (128-141) & $\mathrm{X}$ & $\mathrm{X}$ & $\mathrm{X}$ & \\
\hline S5 & NFTVQELALK (43-52) & $\mathrm{X}$ & $\mathrm{X}$ & & $\mathrm{X}$ \\
\hline
\end{tabular}

-Detected peptide present in source (X-box); Detected peptide absent in source (white box)

-Common peptide (detected among all sources)

-Instruments: MALDI-TOF, LTQ-Orbitrap \& Q-Tof Premier (-) Rapigest

peptides that were detected among Ptx and Ptxd sources analyzed. More importantly, an in-depth visual scan analysis of MS/MS spectra of the common peptides revealed similar fragmentation profiles (based on y- and b-ion distribution and relative intensity of fragment ions), suggestive of Ptx subunit sequence homogeneity as illustrated in Figure 1.
In addition to the determination of sequence homogeneity among sources, the relative amounts of protein as measured by relative abundance (fmol) of subunits from Ptx and Ptxd was preliminarily explored (Table 5). An LCMS/MS approach was used, in which the Q-Tof $\mathrm{MS}^{\mathrm{E}}$ data were employed for this assessment in which differential 
TABLE 3: Comparative pertussis toxin and toxoid subunit sequence analysis ( $-/+$ rapigest treatment).

\begin{tabular}{|c|c|c|c|c|c|}
\hline Subunit & Source & $\begin{array}{c}-\mathrm{RG} \\
(\% \mathrm{AA} \\
\text { coverage) }\end{array}$ & $\begin{array}{c}\# \\
\text { peptides } \\
\text { detected }\end{array}$ & $\begin{array}{c}+\mathrm{RG} \\
(\% \mathrm{AA} \\
\text { coverage) }\end{array}$ & $\begin{array}{c}\text { \# peptides } \\
\text { detected }\end{array}$ \\
\hline \multirow{4}{*}{ S1 } & Toxin A & 58 & 10 & 57 & 10 \\
\hline & Toxin B & 69 & 12 & 74 & 12 \\
\hline & Toxoid C & 49 & 8 & 66 & 10 \\
\hline & Toxoid D & 41 & 7 & 70 & 11 \\
\hline \multirow{4}{*}{ S2 } & Toxin A & 33 & 4 & 31 & 4 \\
\hline & Toxin B & 39 & 4 & 45 & 7 \\
\hline & Toxoid C & 14 & 2 & 14 & 2 \\
\hline & Toxoid D & 5 & 1 & 14 & 2 \\
\hline \multirow{4}{*}{ S3 } & Toxin A & 28 & 5 & 49 & 8 \\
\hline & Toxin B & 39 & 6 & 60 & 9 \\
\hline & Toxoid C & 12 & 2 & 38 & 6 \\
\hline & Toxoid D & 18 & 2 & 33 & 5 \\
\hline \multirow{4}{*}{ S4 } & Toxin A & 53 & 4 & 28 & 2 \\
\hline & Toxin B & 57 & 4 & 41 & 3 \\
\hline & Toxoid C & 28 & 2 & 28 & 2 \\
\hline & Toxoid D & 28 & 2 & 50 & 4 \\
\hline \multirow{4}{*}{ S5 } & Toxin A & - & - & 10 & 1 \\
\hline & Toxin B & - & - & 18 & 2 \\
\hline & Toxoid C & - & - & 10 & 1 \\
\hline & Toxoid D & - & - & 10 & 1 \\
\hline
\end{tabular}

-Approach: Electrospray Ionization Mass Spectrometry-Instrument: LTQOrbitrap only-RG (Rapigest)

abundance was revealed among sources and the subunits. The absolute abundance of Ptx B was 7.2-fold higher among all subunits compared to Ptx A. Ptx B S4 gave the highest fold change at nearly 19 in comparison to Ptx A. With respect to Ptxd, the abundance was nearly the same for all of the subunits, with Ptxd D S1 revealing the highest fold change (1.5) versus Ptxd C.

\section{Discussion}

We initiated a study using mass spectrometry to determine if AA sequence differences exist between Ptx and Ptxd when examined from different sources. Using proteins (i.e., Ptx) from strains that may not share the exact same AA sequence or that have undergone different inactivation treatments could result in differences in immune effectiveness. Protein sequence variation between strains may be small (i.e., single AA addition, deletion, or substitution) or potentially more extensive with the addition or deletion of immunogenically relevant motifs or domain structures. As a result, protein sequence variance could lead to subtle structural, conformational, and/or functional differences that can be detected only through the use of sensitive technologies.

Previous MS investigations have been used for identification, differentiation, and characterization of protein complexes. For example, Nandakumar et al. [22] utilized MS techniques for identification of bacterial proteins. Protein mixtures were subject to tryptic in-solution digestion and analyzed by reverse phase nano LC-MS/MS followed by database analysis. Other investigators have used MALDITOF MS/MS for the analysis of peptides generated from the enzymatic cleavage of Clostridium botulinum C3 coenzyme [23]. Also, Yoder and colleagues analyzed tryptic digests of Vaccinia virus using MALDI-TOF, as well as a quadrupole ion trap mass spectrometer and a quadrupole TOF to identify proteins associated with infection [24]. Additionally, nanoLC-MS/MS was used to identify AA variability between twobovine surfactant proteins [25]. In our study, an analytical and proteomic evaluation was conducted to assess any composition (i.e., AA sequence of the protein components) and formulation (i.e., how much of a specific component) disparities among Ptx and Ptxd.

Based on AA coverages and peptide detection alone, the two Ptxs and two Ptxds appear to have similar sequences, an indication that they were derived from the same origin, although different purification schemes and inactivation methods most likely were employed. The fact that the Ptx and Ptxd sources shared similar tryptic digest patterns, in terms of the peptides being generated and detected as well as spectrum profiles, suggests homogeneity. The presence of RG improved protein digestion resulting in higher-percent coverages generally across source as well as Ptx subunits. In addition, RG treatment appeared to have exposed the smallest-sized S5, resulting in an increase in coverage for this subunit. In doing so, additional peptides were detected that once again were shown to be similar among Ptx and Ptxd sources based on $y$ - and b-ion distribution and relative intensities of the fragmented peptides. Moreover, studies by Sekura et al. [26] revealed, by amino acid analysis, that Ptx purified from Bp strain 165 was indistinguishable from Ptx prepared from the well-characterized Bp Tahoma strain. In addition, Tummala et al. confirmed Ptx sequence homogeneity among their Bp culture supernatant purified Ptx as compared with previously reported sequence information [19]. These data are confirmatory and strengthen the MS results in this study for Ptx sequence homogeneity. However, although the data suggest protein sequence homogeneity, if the sequence is indeed different but not present in the database then, upon searching, the generated peptides would not be detected resulting in a lower peptide map coverage.

Ptx is a major virulence factor and in its natural state has physiological inhibitory effects [11]. The subunits are highly immunoreactive, including the dominant S1, making it an ideal component for vaccines. To reduce the cellular lethality induced by Ptx, the Ptx is inactivated or detoxified in its final vaccine form. Inactivation occurs by chemical modification (i.e., formaldehyde or glutaraldehyde) via the formation of covalent crosslinkings on targeted AA (usually lysines). Interestingly in Ptxd samples, the oligomeric containing subunits percent AA coverage was lower. The S2-S5 subunits, rich in lysine are the subunits that are known to be heavily modified due to the chemical action exhibited by formaldehyde and/or glutaraldehyde [13, 27, 28]. As a result of modification, these peptides and subsequent MS-MS profiles would thus not be 
TABle 4: Peptide detection among pertussis toxin and toxoid subunits (-/+ rapigest treatment).

\begin{tabular}{|c|c|c|}
\hline & $-\mathrm{RG}$ & $+\mathrm{RG}$ \\
\hline \multirow{7}{*}{ S1 } & & GTGHFIGYIYEVR (114-126) \\
\hline & GTGHFIGYIYEVR (114-126) & YTEVYLEHR (93-101) \\
\hline & YTEVYLEHR (93-101) & MQEAVEAER (102-110) \\
\hline & MQEAVEAER (102-110) & IPPENIR (170-176) \\
\hline & IPPENIR (170-176) & VYHNGITGETTTTEYSNAR (181-199) \\
\hline & & ILAGALATYQSEYLAHR (152-168) \\
\hline & & MAPVIGACMAR (228-238) \\
\hline \multirow{2}{*}{ S2 } & \multirow{2}{*}{ GSGDLQEYLR (64-73) } & GSGDLQEYLR (64-73) \\
\hline & & NTGQPATDHYYSNVTATR (120-137) \\
\hline \multirow{4}{*}{ S3 } & \multirow{4}{*}{ ALFTQQGGAYGR (39-50) } & ALFTQQGGAYGR (39-50) \\
\hline & & QITPGWSIYGLYDGTYLGQAYGGIIK (75-100) \\
\hline & & GNAELQTYLR (65-74) \\
\hline & & DAPPGAGFIYR (101-111) \\
\hline \multirow{2}{*}{ S4 } & \multirow{2}{*}{ TNMVVTSVAMKPYEVTPTR (51-69) } & TNMVVTSVAMKPYEVTPTR (51-69) \\
\hline & & RPGSSPMEVMLR (100-111) \\
\hline S5 & & NFTVQELALK (43-52) \\
\hline
\end{tabular}

-Peptides detected in +RG (rapigest) (in bold) -Approach: Electrospray Ionization Mass Spectrometry-Instrument: LTQ-Orbitrap only

TABle 5: Comparative pertussis toxin and toxoid subunit quantitative analysis.

\begin{tabular}{lcccccc}
\hline Source & S1 & S2 & S3 & S4 & S5 & Total (all subunits) \\
\hline Toxin A & 0.38 & 0.19 & 0.13 & 0.02 & 0.09 & 0.81 \\
Toxin B & 1.48 & 2.04 & 1.35 & 0.37 & 0.57 & 5.81 \\
Toxoid C & 1.88 & 0.34 & 0.32 & ND & ND & 2.54 \\
Toxoid D & 2.91 & 0.40 & 0.21 & ND & 0.06 & 3.54 \\
\hline
\end{tabular}

-Measured as relative abundance $(\mathrm{f} \mathrm{mol} / \mu \mathrm{L})$

-ND: not detected

-Approach: Electrospray Ionization Mass Spectrometry

-Instrument: Q-Tof Premier only

detected using traditional selected database searching tools resulting in lower AA coverages.

Although formaldehyde, for instance, is an ideal reagent for chemical crosslinking, it also immobilizes the antigen (i.e., Ptx) and alters its conformation preventing a natural interaction between the antibody and antigen. In other words, formaldehyde reduces the accessibility of antibodies to their epitopes by creating steric hindrance or by altering the epitope site itself. In Ptxd samples, inactivation by formaldehyde appears to have a severe impact on peptide detection, due to crosslinking of lysine on the Ptx subunits $[13,27-29]$. These subunits are most likely masked preventing trypsin, whose natural recognition sites are lysine and arginine, to effectively cleave the protein resulting in a lower AA coverage. On a cellular level, it is sensible for these subunits to be inactivated heavily, since in vivo, they make up the oligomeric complex that adheres to the human host cells which upon binding to the appropriate receptor initiates Ptx function. Blocking these cellular activities, in essence, inhibits or diminishes further downstream enzymatic activity by S1, which would allow for progression of normal immune responses by the host towards Bp. Besides, S1 has consistently had higher AA coverage reporting among all sources, which could be explained by the fact that $S 1$ lacks lysines. Although S1 is deficient in lysine, free cellular lysine in the presence of formaldehyde would still create crosslinking bridges that lead to the detoxification $[28,29]$ but to a lesser extent compared to S2-S5. As a result of minimal chemical bombardment and thus potentially incomplete inactivation by formaldehyde on the toxic S1 component, residual toxicity may remain, with varying levels among Ptxd sources, could lead and be an explanative factor for differential immunity, albeit speculative.

\section{Conclusions}

In conclusion, this study incorporated multiple massspectrometric approaches to identify protein sequence similarities among Ptx and Ptxd sources, based on AA coverage profiles, peptide detection, and fragmentation profiles. Lower-percent coverage among Ptxd oligomeric subunits as compared to S1 could be explained most likely due to chemical modifications. As a result of these modifications, inactivation of Ptx subunits, as evident by failed detection of peptides, could lead to diminished immuno-cellular interactions between human-generated antibodies against chemically modified $\mathrm{Bp}$ antigens. In addition to the protein components, changes in the formulation, including the concentration, ratio and abundance of the Ptx subunits could translate into differences with respect to immune responses and vaccine efficacy. Moreover, information from this MS study could prove useful in improving immunological tests that use Ptx as an examining factor for vaccine evaluation and even pertussis diagnosis.

Future studies such as de novo sequencing or protein sequence tag analysis may be considered to validate and 
strengthen the current finding that the protein sequences among all the sources are indeed homogeneous. In addition, incorporation of other enzymes in the presence of RG to expand the AA coverage and peptide detection may also be performed. Also, a comparative quantitative MS analysis assessing the functionality of Ptx and Ptxd sources may be able to determine the effect, if any, that Ptx inactivation, Ptx function, and potential residual toxicity play on vaccine efficacy such as immune responses. Lastly, in vivo animal studies may be conducted to examine the correlation between Ptx protein sequence homogeneity and immunoreactivity.

\section{Disclaimer}

The references in this paper to any specific commercial products, process, service, manufacturer, or company do not constitute an endorsement or a recommendation by the U.S. Government or the Centers for Disease Control and Prevention. The findings and conclusions in this paper are those of the authors and do not necessarily represent the views of CDC.

\section{References}

[1] M. Singh and K. Lingappan, "Whooping cough: the current scene," Chest, vol. 130, no. 5, pp. 1547-1553, 2006.

[2] J. B. Bobbins, R. Schneerson, B. Trollfors, et al., "The diphtheria and pertussis components of diphtheria-tetanus toxoids-pertussis vaccine should be genetically inactivated mutant toxins," The Journal of Infectious Diseases, vol. 191, no. 1, pp. 81-88, 2005.

[3] Q. He and J. Mertsola, "Factors contributing to pertussis resurgence," Future Microbiology, vol. 3, no. 3, pp. 329-339, 2008.

[4] World Health Organization, "WHO Recommended Standards for Surveillance of Selected Vaccine-preventable diseases," WHO/V\&B/02.01, 2003.

[5] Centers for Disease Control and Prevention, "Pertussis vaccination: use of acellular pertussis vaccines among infants and young children: recommendations of the Advisory Committee on Immunization Practices (ACIP)," Morbidity and Mortality Weekly Report, vol. 46, no. RR-7, pp. 1-25, 1997.

[6] K. Kretsinger, K. R. Broder, M. M. Cortese, et al., "Preventing tetanus, diphtheria, and pertussis among adults: use of tetanus toxoid, reduced diphtheria toxoid and acellular pertussis vaccine recommendations of the Advisory Committee on Immunization Practices (ACIP) and recommendation of ACIP, supported by the Healthcare Infection Control Practices Advisory Committee (HICPAC), for use of Tdap among health-care personnel," Morbidity and Mortality Weekly Report, vol. 55, no. RR-17, pp. 1-37, 2006.

[7] K. R. Broder, M. M. Cortese, J. K. Iskander, et al., "Preventing tetanus, diphtheria, and pertussis among adolescents: use of tetanus toxoid, reduced diphtheria toxoid and acellular pertussis vaccines recommendations of the Advisory Committee on Immunization Practices (ACIP)," Morbidity and Mortality Weekly Report, vol. 55, no. RR-3, pp. 1-34, 2006.

[8] K. R. Powell, R. S. Baltimore, H. H. Bernstein, et al., "Prevention of pertussis among adolescents: Recommendations for use of tetanus toxoid, reduced diphtheria toxoid, and acellular pertussis (Tdap) vaccine," Pediatrics, vol. 117, no. 3, pp. 965978, 2006.

[9] B. Taylor and R. Fahim, "Pasteur Merieux Connaught fivecomponent acellular pertussis vaccine," Biologicals, vol. 27, no. 2, pp. 103-104, 1999.

[10] C. Locht, R. Antoine, and F. Jacob-Dubuisson, "Bordetella pertussis, molecular pathogenesis under multiple aspects," Current Opinion in Microbiology, vol. 4, no. 1, pp. 82-89, 2001.

[11] M. Tamura, K. Nogimori, S. Murai, et al., "Subunit structure of islet-activating protein, pertussis toxin, in conformity with the A-B model," Biochemistry, vol. 21, no. 22, pp. 5516-5522, 1982.

[12] J. Moss and M. Vaughan, "Activation of adenylate cyclase by choleragen," Annual Review of Biochemistry, vol. 48, pp. 581600, 1979.

[13] S. Fowler, D. K.-L. Xing, B. Bolgiano, C.-T. Yuen, and M. J. Corbel, "Modifications of the catalytic and binding subunits of pertussis toxin by formaldehyde: effects on toxicity and immunogenicity," Vaccine, vol. 21, no. 19-20, pp. 2329-2337, 2003.

[14] E. S. Bamberger and I. Srugo, "What is new in pertussis?" European Journal of Pediatrics, vol. 167, no. 2, pp. 133-139, 2008.

[15] J. O. Lay Jr., "MALDI-TOF mass spectrometry of bacteria," Mass Spectrometry Reviews, vol. 20, no. 4, pp. 172-194, 2001.

[16] C. Fenselau and F. A. Demirev, "Characterization of intact microorganisms by MALDI mass spectrometry," Mass Spectrometry Reviews, vol. 20, no. 4, pp. 157-171, 2001.

[17] G. Chen and B. N. Pramanik, "LC-MS for protein characterization: current capabilities and future trends," Expert Review of Proteomics, vol. 5, no. 3, pp. 435-444, 2008.

[18] D. Bottero, M. E. Gaillard, M. Fingermann, et al., "Pulsed-field gel electrophoresis, pertactin, pertussis toxin S1 subunit polymorphisms, and surfaceome analysis of vaccine and clinical Bordetella pertussis strains," Clinical and Vaccine Immunology, vol. 14, no. 11, pp. 1490-1498, 2007.

[19] M. Tummala, P. Hu, S.-M. Lee, A. Robinson, and E. Chess, "Characterization of pertussis toxin by LC-MS/MS," Analytical Biochemistry, vol. 374, no. 1, pp. 16-24, 2008.

[20] A. Keller, A. I. Nesvizhskii, E. Kolker, and R. Aebersold, "Empirical statistical model to estimate the accuracy of peptide identifications made by MS/MS and database search," Analytical Chemistry, vol. 74, no. 20, pp. 5383-5392, 2002.

[21] A. I. Nesvizhskii, A. Keller, E. Kolker, and R. Aebersold, "A statistical model for identifying proteins by tandem mass spectrometry," Analytical Chemistry, vol. 75, no. 17, pp. 46464658, 2003.

[22] R. Nandakumar, N. Madayiputhiya, and A. F. Fouad, "Proteomic analysis of endodontic infections by liquid chromatography-tandem mass spectrometry," Oral Microbiology and Immunology, vol. 24, no. 4, pp. 347-352, 2009.

[23] M. V. Muetzelburg, F. Hofmann, I. Just, and A. Pich, "Identification of biomarkers indicating cellular changes after treatment of neuronal cells with the C3 exoenzyme from Clostridium botulinum using the iTRAQ protocol and LCMS/MS analysis," Journal of Chromatography B, vol. 877, no. 13, pp. 1344-1351, 2009.

[24] J. D. Yoder, T. S. Chen, C. R. Gagnier, S. Vemulapalli, C. S. Maier, and D. E. Hruby, "Pox proteomics: mass spectrometry analysis and identification of Vaccinia virion proteins," Virology Journal, vol. 3, article 10, 2006. 
[25] S. Liu, L. Zhao, D. Manzanares, et al., "Characterization of bovine surfactant proteins $\mathrm{B}$ and $\mathrm{C}$ by electrospray ionization mass spectrometry," Rapid Communications in Mass Spectrometry, vol. 22, no. 2, pp. 197-203, 2008.

[26] R. D. Sekura, F. Fish, C. R. Manclark, B. Meade, and Y. L. Zhang, "Pertussis toxin. Affinity purification of a new ADPribosyltransferase," Journal of Biological Chemistry, vol. 258, no. 23, pp. 14647-14651, 1983.

[27] M. J. Corbel, D. K. L. Xing, B. Bolgiano, and D. J. Hockley, "Approaches to the control of acellular pertussis vaccines," Biologicals, vol. 27, no. 2, pp. 133-141, 1999.

[28] J. B. Robbins, R. Schneerson, J. M. Keith, J. Shiloach, M. Miller, and B. Trollors, "The rise in pertussis cases urges replacement of chemically-inactivated with genetically-inactivated toxoid for DTP," Vaccine, vol. 25, no. 15, pp. 2811-2816, 2007.

[29] C. Montero, "The antigen-antibody reaction in immunohistochemistry," The Journal of Histochemistry and Cytochemistry, vol. 51, no. 1, pp. 1-4, 2003. 

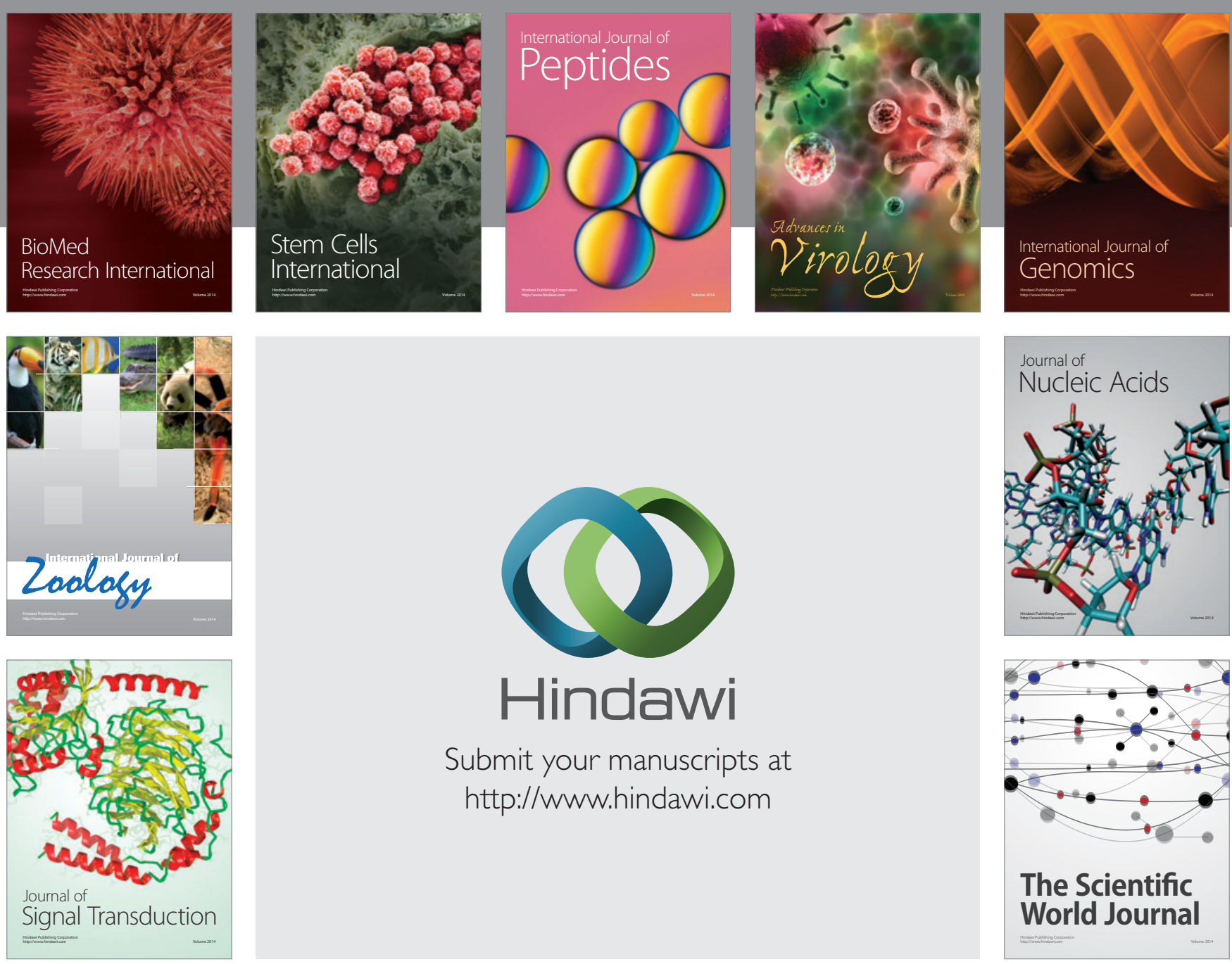

Submit your manuscripts at

http://www.hindawi.com
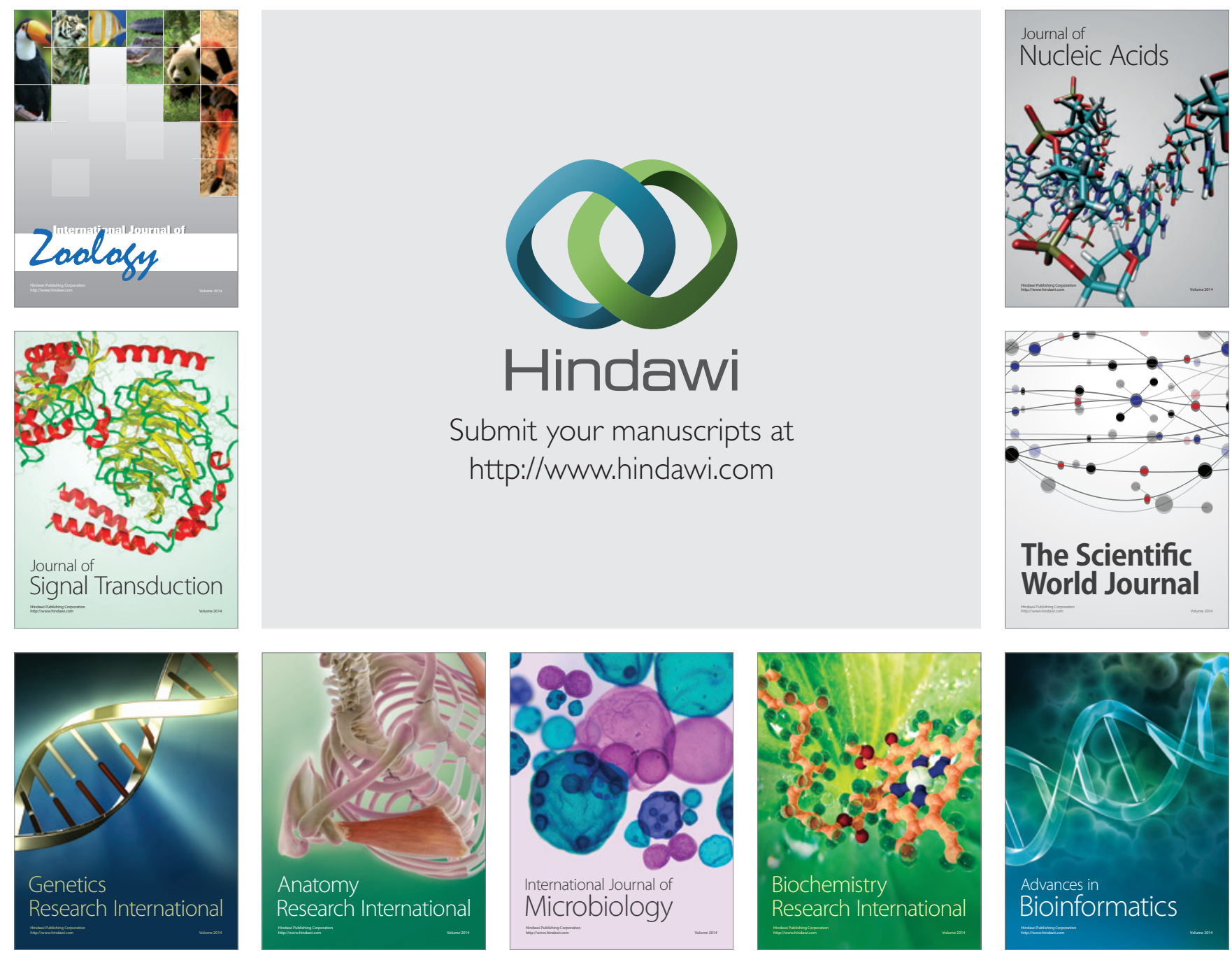

The Scientific World Journal
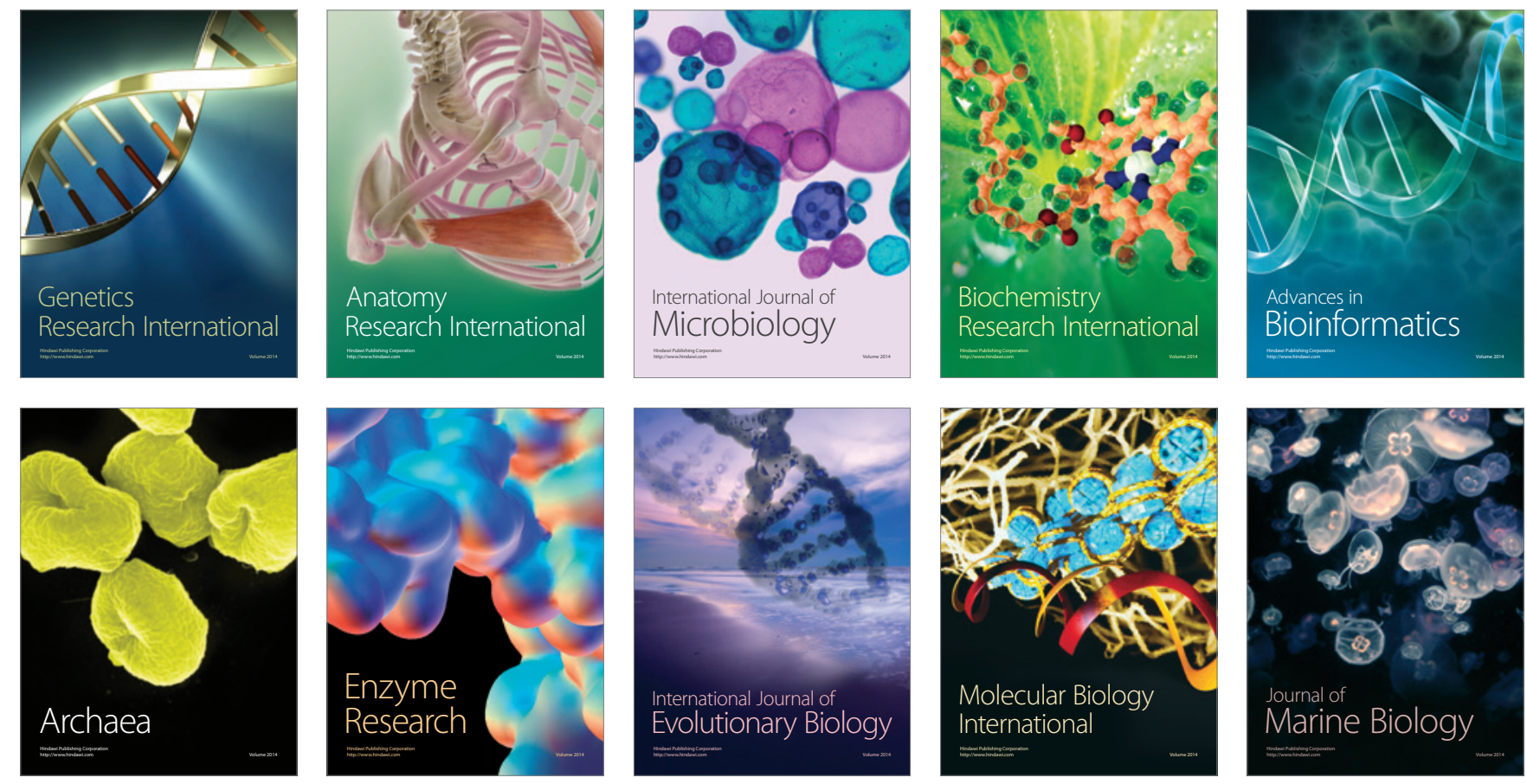